\title{
LAS CONCEPCIONES DEL ESPACIO EN EL DICTIONNAIRE DE BAYLE
}

\author{
Albert RIBAS \\ Universidad Oberta de Catalunya
}

\section{Introducción}

Desde hace ya unas décadas ha dejado de tomarse la figura de Pierre Bayle (1647-1706) como simple precursor del espíritu ilustrado del siglo XVIII. Esa apropiación anacrónica de Bayle ha sido en parte desalojada en beneficio de otra aproximación: la de tomarlo y comprenderlo en su propio contexto -aun reconociendo su importantísimo papel en el desarrollo de las ideas que han de conducir a la Ilustración ${ }^{1}$.

Por lo tanto, hay que tomar su Dictionnaire historique et critique ${ }^{2}$ sobre todo como una fuente privilegiada de información acerca de las ideas debatidas en su época. Y también hay que tomarlo como síntoma de la lucha, por una parte, entre el espíritu de sistema propio del racionalismo y, por otra, la reivindicación de una razón en minúsculas, una razón modesta que huye de las grandes construcciones y prefiere centrarse en el examen a menudo contradictorio de las posiciones confrontadas 3 . Para este examen, el método es el "histórico y

1 Véase al respecto, por ejemplo, Pierre RETAT, Le Dictionnaire de Bayle et la lutte philosophique au xvitieme sizcle (Vrin, Paris, 1959), pp. 7-12.

2 La primera edición del Dictionnaire es de 1697; la segunda, corregida y aumentada, de 1702. Esta segunda edición aporta lo básico de los contenidos del Dictionnaire tal como aparece en las siguientes ediciones, hasta alcanzar a la onceava de 1820-1824. En lo sucesivo, el Dictionnaire será citado por la edición más asequible, la reimpresión facsímil de1969: Pierre BAYLE, Dictionnaire historique et critique, 16 tomos, Paris, 1820-1824 (reimpr. facs. Slatkine Reprints, Ginebra, 1969). Para la consulta de los artículos manejados aquí, esta edición de 1820-1824 es adecuada pues su contenido no difiere del correspondiente a la $2 .^{a}$ edición de 1702.

3 Cfr. Alexandre KoYrE, De la mystique d̀ la science (ed. p. Redondi, Eehess, París, 1986), pp. 91-2 y 101-2. 
crítico" - tal como recoge el título completo del Dictionnaire-, o sea el llevar a cabo una recapitulación de los argumentos en presencia remontándose a sus antecedentes históricos.

Tal consideración del Dictionnaire como fuente válida para conocer el debate de ideas en el umbral de los siglos XVII y XVIII es, por añadidura, el más pertinente con respecto al tema que nos ocupa, es decir el de las concepciones del espacio. Los contenidos del Dictionnaire referidos a esta cuestión son, en efecto, un muy buen testimonio de una época que hemos de considerar a modo de encrucijada. Recordemos, por ejemplo, que esa época es la inmediatamente anterior a la polémica Leibniz-Clarke (1715-1716), o sea la polémica entre espacio absoluto y espacio relativo, y también entre espacio pleno y espacio vacío. Es una época de encrucijada entre la concepción cartesiana y la newtoniana, con otras importantes referencias a las influencias del atomismo de un Gassendi, a la mencionada posición de Leibniz, a la elaboración de Locke. En suma, es el inmediato antecedente de la escisión vivida en la opinión filosófica europea acerca del espacio, escisión que Voltaire unos años después se encargará de expresar en su famosa Catorceava Carta - al decir que en Inglaterra se concibe un espacio vacío mientras en el continente se concibe el pleno

Los contenidos del Dictionnaire ilustran y aclaran, aunque sea de modo disperso y parcial, estas diversas interpretaciones. Básicamente, la reseña y discusión de Bayle se centra en estos dos aspectos: por una parte, en la discusión sobre la posibilidad o la consistencia de que tal espacio sea vacío; por otra parte, en el estatus ontológico del espacio, con una interesante apelación a la concepción del espacio - tomada de Locke - como entidad relativa al entendimiento. Esta discusión viene acompanada de múltiples referencias al aspecto teológico del problema, o sea a la posible correlación entre espacio e inmensidad divina. Como se verá, además, de este conjunto de consideraciones también podrá deducirse una opinión sobre el problema de la geometrización y la divinización del espacio como factores inscritos en el proceso de la conceptualización moderna del espacio.

En resumen, el Dictionnaire de Bayle se nos presenta como un buen testimonio de la lucha de ideas en torno a las concepciones del espacio en el um-

4 Dirá Voltaire en 1733: "Un Français qui arrive à Londres trouve les choses bien changées en Philosophie comme dans tout le reste. Il a laissé le monde plein, il le trouve vide; à Paris on voit l'Univers composé de tourbillons de matière subtile; à Londres on ne voit rien de cela..." (Voltaire, Lettres philosophiques, $14 .^{2}$ carta, ed. G. Lanson, París, 1909, vol. 2, p. 1). 
bral de los siglos XVII y XVII, lucha que Bayle documenta en las tres vertientes que han de tomarse en cuenta: la vertiente científica, la filosófica y la teológica.

\section{Clasificación de contenidos}

Si consultamos el índice temático del Dictionnaire, sólo hallamos dos referencias directas al espacio, con las siguientes aclaraciones:

Espace. Si ce n'est autre chose que l'immensité de Dieu. XV. 54.

Espaces imaginaires. Suarez tâche d'expliquer comment Dieu peut y être présent. VI. $588^{5}$.

La primera mención remite al artículo sobre Zenón de Elea ("Zénon d'Élée"); la segunda al artículo sobre Sebastian Franck ("Francus"). Y como vemos, el carácter de la discusión es aquí básicamente en términos teológicos.

Junto a estas dos referencias, consultando otros apartados del índice temático-por ejemplo, atomes, vide, étendue, matière, mathématiques, immensité-, y cruzando las referencias entre sí, observamos que la mayor parte de las remisiones conducen sólo a unos pocos artículos más: principalmente al artículo sobre Leucipo ("Leucippe"), y en menor grado a los artículos "Pascal", "Spinoza", y algunos otros que ya serán comentados oportunamente.

De hecho, para una visión de conjunto de la exposición de Bayle, prácticamente bastan los artículos "Zénon" y "Leucippe". A modo de resumen, puede decirse que el artículo "Zénon" aborda la cuestión más específicamente filosófica, o sea el problema ontológico del espacio; y que el artículo "Leucippe" aporta una discusión más concreta sobre el problema del espacio vacío.

Pero debe advertirse que en estos artículos, al igual que en los restantes, la exposición no es en absoluto sistemática: los temas se cruzan y se mezclan, obligando a nuevas consultas. Por lo tanto, al proponer una clasificación temática de los contenidos del Dictionnaire, ha de tenerse presente esta característica. De hecho, esta falta de sistematicidad de Bayle proviene del hecho que su Dictionnaire no se organiza por temas, sino por personajes. Y obedece además al talante de Bayle, que no pretende exponer una verdad, sino un examen de las diversas verdades enunciadas, mostrando las dificultades que contiene cada una de ellas y sometiéndolas al contraste y a la contradicción con sus opuestas.

5 Dictionnaire, tomo XVI, p. 403. 
Hechas estas precisiones, proponemos la siguiente clasificación temática:

i. La consideración científico-experimental, especialmente a propósito de los experimentos del vacío.

ii. La consideración propiamente filosófica, o sea los problemas ontológicos en las concepciones del espacio.

iii. La consideración teológica, traída a través del tema de la relación entre espacio e inmensidad de Dios.

Cabría añadir, además, que los puntos ii y iii, al apuntar al problema de la equivalencia entre Dios y extensión, conectan con otro importante tema -recurrente en Bayle-, esto es, la cuestión del espinosismo.

\section{Sobre el vacío experimental}

El aspecto científico-experimental de la cuestión del espacio se centra básicamente en esta cuestión del vacío. Pues, en efecto, la aceptación o no del vacío incide en un punto clave de la cuestión del espacio: si se admite el vacío, quiere decirse que espacio y cuerpo son entidades distintas, que es aceptable una extensión libre de materia; por el contrario, si no se admite el vacío considerándolo una imposibilidad en sí misma, se refuerza la opinión de que espacio y cuerpo son entidades no separadas. Esta segunda opción es, por ejemplo, la de Descartes al señalar la obligatoria correlación entre espacio y cuerpo; es lo que se afirma al caracterizar la materia como res extensa.

Esta discusión, que se remonta ya a la Antigüedad —con la confrontación entre la posición antivacuista de Aristóteles y la posición vacuista del atomismo-, adquiere una extraordinaria actualidad en el siglo XVII. Como es sabido, en este siglo irrumpen nuevos argumentos vacuistas con el diseño y realización de nuevos experimentos: son fundamentalmente los experimentos de Torricelli, de Pascal, de Guericke, de Boyle, experimentos que parecen probar el vacío y que han de tomarse como los antecedentes empíricos de lo que Newton sistematizará en su nueva cosmología de los inmensos espacios vacíos. Entre los experimentos barométricos de Torricelli (de 1644) y la publicación por Newton de sus Principia (1687) habría que situar esa etapa de la demostración empírica del vacío ${ }^{6}$. Sin embargo, esas nuevas aportaciones experimentales no se

6 Este episodio ya ha sido bien estudiado. Véase por ejemplo: Cornelis DE WARD, L'experience barométrique, ses antécédents et ses explications. Etude historique (Imprimerie Nouvelle, 
impusieron sin resistencia. Todo el siglo XVII es testigo de esa ardua batalla entre los partidarios del vacío y sus detractores. En el campo de los primeros - los partidarios del vacío- las posiciones no son homogéneas, pues incluyen desde los más radicales en la afirmación del vacío (caso de Guericke) hasta los más pragmáticos (caso de Boyle); en el campo de los detractores hay que incluir a los filósofos más influyentes, con Descartes a la cabeza y siguiendo con Hobbes, Spinoza, Leibniz, y la larga lista de todos aquellos que se adscriben al saber transmitido por la tradición escolástica.

Así pues, en el cambio del siglo XVI a XviI la discusión vacuista no es en absoluto un debate cerrado, al contrario. Lo confirmará en el 1715-1716 la polémica entre Leibniz y el newtoniano Clarke, y lo confirma el peso todavía importante de la opinión cartesiana.

En este contexto, sorprende en principio que en el Dictionnaire de Bayle las referencias al vacío experimental sean más bien escasas. Básicamente las hallamos en los artículos "Pascal", "Magni", y "Leucippe"7, pero sin una exposición detallada ni exhaustiva de la historia de los experimentos científicos. Incluso ocupa más espacio la relación de las discusiones sobre la cuestión de la autoría de los experimentos —así la acusación de plagio dirigida a Magni ${ }^{8}$ - que la descripción de éstos.

Bayle se limita a señalar las dos conclusiones principales que se desprenden de los experimentos de Torricelli y de Pascal: la caducidad del viejo principio del horror vacui y el papel del peso del aire en ellos. Al margen de estas dos re-

Thouars, 1936); Steven SHAPIN y Simon SCHAFFER, Leviathan and the air-pump. Hobbes, Boyle, and the experiemental life (Princeton, 1985); Albert RIBAS, Biografia del vacio (tesis doctoral, Universidad de Barcelona, 1995), capítulo II.I.

7 En el artículo "Pascal" (tomo XI, 419-437a) sólo es abordado - sobre un total de nueve notas- en la nota F (Après avoir travaille... aux expériences de la nouvelle philosophie, il abandonna cette étude. 424b-427b). En el artículo "Magni" (tomo X, 50a-53b), se aborda en la nota $\mathrm{B}$ (On prétend qu'il se voulut attribuer l'invention... de Torricelli. $51 \mathrm{ab}$ ). En el artículo "Leucip-

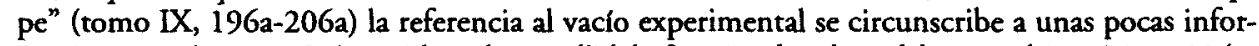
maciones en la nota $\mathrm{G}$ (Le vide... devient lidole favorite des plus cellebres mathematiciens. 204a206a); la discusión es aquí fundamentalmente doctrinal.

8 Aparece en la citada nota B del art. "Magni" y en el art. "Pascal", 426. Fue sobre todo Roberval y el biógrafo de Descartes - Baillet - quienes acusaron al franciscano Valeriano Magni de plagiar a Torricelli y a Pascal. La obra de Valeriano MAGNI, Admiranda de Vacuo (Varsovia, 1647), sin embargo, es anterior cuando menos a las de Pascal, lo cual contradice las pretensiones críticas hacia Magni. Sobre este conflicto de atribuciones, véase Alexandre KoYRE, "Pascal savant", en Etudes d'histoire de la pensée scientifique (Gallimard, París, 1973), pp. 362-389. 
ferencias y de la cuestión de los plagios, no hay más información sobre la vertiente experimental: por ejemplo, se echa en falta alguna explicación sobre los famosos experimentos de Guericke, los de Boyle, o al menos los desarrollados en París en el ámbito de las actividades de la Académie Royale de Sciences en las últimas décadas del siglo. $Y$ es que el aspecto científico y experimental del vacío interesa poco a Bayle.

Este enfoque tan reducido nos sugiere cúal era el peso de la vertiente experimental en el debate vacuista del cambio de siglo, un peso ciertamente limitado a pesar de la unanimidad sobre las dos conclusiones ya reseñadas: no al horror vacui y papel del peso del aire. Esta unanimidad no impide la discusión sobre los fundamentos metafísicos de la aceptación o no del vacío -que es la discusión que interesa de verdad a Bayle - ni anula las objeciones cartesianas a la afirmación del vacío. Bayle se encarga de recordar por ejemplo —en el artículo "Pascal" 9- cómo Descartes opinaba que tales experimentos eran conformes a sus principios antivacuistas (con su explicación basada en la materia sutil). En una palabra, a los cartesianos y a otros adversarios del vacío las pruebas empíricas no les hicieron cambiar de opinión ${ }^{10}$. $Y$ así, la polémica no puede circunscribirse a esta vertiente experimental, y ha de derivar forzosamente hacia otros contenidos y otras correlaciones de carácter más doctrinal. El Dictionnaire muestra la amplitud de esas correlaciones.

El testimonio de Bayle en esta vertiente científico-esperimental muestra que su intención es la de subrayar las citadas contradicciones entre la interpretación vacuista y la plenista. Y su opinión personal es la de mostrarse contrario al vacío, sobre todo porque no está de acuerdo con la tesis del carácter del espacio como entidad independiente. Sin embargo, Bayle en su exposición da fe de que la interpretación vacuista se impone en los ámbitos científicos. Así lo dice en la nota $\mathrm{G}$ del citado artículo "Leucippe":

9 Art. "Pascal" (tomo XI, 425b-426). También aquí se reivindica para esos experimentos la paternidad de Descartes, designándolos como la "expérience de Descartes". Bayle (cf. 425b) se apoya, citándolo, en el biografo de Descartes, Adrien Baillet, La vie de Monsieur Descartes (París, 1691), vol. II, p. 330.

10 Téngase en cuenta, por ejemplo, un tratado como el del cartesiano Jacques RoHaulT, Traité de physique (París, 1671). Especialmente en I, caps. 8 y 12, da cuenta de los experimentos barométricos bajo una interpretación plenista. Este Traité de Rohault será la gran referencia de la fisica cartesiana y su vigencia se prolonga hasta entrado el siglo xviI, o sea hasta la victoria newtoniana. Su declive se inicia a partir de su edición inglesa de 1697, edición a cargo del newtoniano Samuel Clarke. 
Disons aussi que le vide, que Gassendi avait rétabli, et que Descartes avait renversé, gagne peu à peu le dessus, et devient l'idole favorite des plus célèbres mathématiciens ${ }^{11}$.

Esta nota G, sobre la que habrá de volver luego, explica, pues, que la opinión vacuista gana terreno sobre la opuesta; y - punto importante- tal opinión es la defendida por los mathématiciens, término que hoy traduciríamos por 'científicos'. Es decir, a pesar de las dificultades filosóficas y doctrinales subrayadas por Bayle, éste reconoce que los principales científicos se adhieren a la idea del vacío (Bayle la llama aquí "ídolo"), y, por lo tanto, a la idea de un espacio independiente que en cuanto tal es vacío.

Como representativos de esta opción, se cita a Torricelli, Gassendi, Huygens y Newton. Y a efectos de resumen, Bayle sitúa la confrontación entre, por una parte, Gassendi, vacuista y atomista que retoma a Leucipo, y, por otra, Descartes, representante del plenismo y heredero de Aristóteles. Pero es a Huygens y a Newton a quienes se refiere al hablar de los mathématiciens. de Newton recuerda su opinión de que el espacio vacío es mucho mayor que el espacio lleno, o sea que la materia es una parte muy pequeña comparada con el espacio total 12; de Huygens afirma que éste se declaró también partidario del vacio, dando a entender que lo hace en unos términos equivalentes a los de Newton -lo cual no es cierto ${ }^{13}$ - Este error a propósito de Huygens puede entenderse acudiendo a la explicación del propio Bayle al final de esta nota $\mathrm{G}$. Ahí se dice que los mathématiciens vacuistas distinguen entre una extensión real (que puede estar ocupada por cuerpos) y el espacio abstracto que es vacío. En esa segunda condideración, en efecto, estarían de acuerdo Newton y Huygens.

Todo ello subraya que el punto de vista que Bayle retiene de los mathématiciens - y es el aspecto que verdaderamente le interesa - es que la visión cien-

11 Tal es el título completo de la nota G del artículo "Leucippe", en $196 \mathrm{ab.}$

12 Cfr. "Leucippe", 205a, citando los Philosophiae Naturalis Principia Mathematica de Isaac Newron (1687). Otras referencias a Newton en 206a del mismo artículo; en "Zénon", 53b; y en "Ovide", 298b. No hemos encontrado otras referencias en el Dictionnaire. de hecho, no hay en el índice temático entrada para Newton.

13 Las referencias a Huygens son prácticamente las mismas que las de Newton: "Leucippe", 205a; "Zénon", 53b. Esta coincidencia subraya la mencionada supuesta identidad entre la concepción de Huygens y la de Newton. En realidad, Huygens no admite la atracción gravitatoria a través del vacío - que es la posición de Newton-, pero no tiene objeción de principio a la posibilidad de un espacio vaciado de materia. Dicho en términos aristotélicos, Huygens admitiría un vacío en potencia, pero no en acto. 
tífica (matemática) se inclina por la consideración del espacio como espacio abstracto, geométrico, y que, en cuanto tal, ese espacio es vacío. Le interesan menos las opiniones sobre la disposición efectiva de la materia en el espacio (si esa materia lo llena todo o no); por eso, el aspecto experimental del vacío o las opiniones científicas sobre esa disposición de la materia retienen poco la atención de Bayle.

En definitiva, tal como ya hemos anunciado, la discusión que interesa a Bayle es la discusión de principios, o sea los aspectos metafísicos y teológicos de la cuestión del espacio, remitiéndola a sus antecedentes históricos.

\section{El problema ontológico del espacio}

Para el examen de esta cuestión, las dos principales referencias son la citada nota G del artículo "Leucippe" y la nota I del artículo "Zénon" 14. Otras referencias menores son los artículos "Simonide", "Spinoza", "Epicure", "Ovide" 15.

Como ya se ha dicho, la nota $G$ del artículo "Leucippe" explica que la hipótesis vacuista gana sobre la opuesta en los medios científicos. Bayle ejemplifica esta confrontación en Gassendi y Descartes, en cuanto son los protagonistas modernos, pero de hecho retrotrae esa discusión a sus antecedentes antiguos. Esto es, la discusión remite a los términos ya planteados por el atomismo clásico. Pues, en efecto, tanto la hipótesis vacuista, como en general la concepción del espacio como entidad independiente, se apoyan en la adaptación de los postulados de la corriente atomista.

Así pues, toda una línea de ataque de Bayle discurrirá en torno a la crítica del atomismo. Esta crítica se desarrolla principalmente en los mencionados ar-

14 Nota G (Le vide... devient l'idole favorite des plus célebres mathématiciens) de "Leucippe" en tomo IX, 204-206; nota I (Je trouves trés apparent qu'il n'oublia les objections que l'on peut fonder sur la distinction du plein et du vide) de "Zénon" en tomo XV, 52-57.

15 Para "Simonide" (tomo XIII), principalmente 297; para "Spinoza" (tomo XIII), principalmente nota $\mathrm{N}$ (La plus monstrueuse hypotbèse... La plus diamétralement opposé aux notions les plus évidentes de notre esprit), 438-446; para "Epicure" (tomo VI), principalmente nota F (Ce fut gâter le systeme des atomes... que de ne pas retenir la doctrine de Démocrite touchant l'âme des atomes), 178, y nota $S$ (Quelques apologistes d'Épicure auraient dû s'efforcer de montrer que son impiété coulait naturellement... de l'existence éternelle de la matière), 191-196; para "Ovide" (tomo XI), principalmente nota G (J'examinerai si les idées des anciens qui ont parlé du chaos, ont été justes, et s'ils on pu dire que cet état ne subsistait plus), 293-302. 
tículos "Leucippe" y "Épicure"; el argumento general consiste en retomar la secular asociación que entre atomismo y ateísmo ha venido estableciéndose. Bayle se explaya en los conocidos argumentos sobre el movimiento azaroso de los átomos, en la falta de un designio divino en ese azar, en el argumento acerca de la eternidad de la materia que supuestamente el sistema atomista avala. Ese ateísmo y materialismo son advertencias suficientemente potentes para desanimar toda apelación al atomismo, apelación que parece subyacente a toda concepción moderna del espacio como espacio independiente de los cuerpos (el espacio-intervalo entre átomos) y como espacio conceptual o fácticamente vacío ${ }^{16}$.

Otras líneas de ataque de Bayle son las siguientes: el examen del esquema 'sustancia / accidente' aplicado al espacio; el examen del argumento clásico acerca de que el movimiento implica el vacío; la calificación del espacio como "ídolo". Todo ello, como veremos, se dirige a dos conclusiones: en primer lugar, la defensa de la acatalepsia (o incomprensibilidad de las cosas); en segundo lugar, la remisión al entendimiento del problema del espacio. Veamos con algún detalle estas líneas argumentativas.

En cuanto al primer punto, o sea el esquema 'sustancia / accidente', el argumento de Bayle parte de la distinción entre el concepto de extensión en el caso de que ésta sólo pueda ser una cualidad de los cuerpos y el concepto de extensión en el caso de considerarla independiente de los cuerpos; en una palabra, los casos del espacio-cuerpo y del espacio vacío. Las nociones que definen la extensión en el primer caso serían la divisibilidad, la movilidad y la impenetrabilidad; por el contrario, en el segundo caso serían la indivisibilidad, la inmovilidad y la penetrabilidad. Y entonces se pregunta Bayle: ese espacio vacío que es la extensión inmóvil, indivisible y penetrable, ¿es sustancia o modo? Si es modo, no lo sería de ninguna sustancia. Si es sustancia, o será creada o increada. Si es creada, sería destruible, lo cual conduciría al absurdo de cuerpo sin extensión. Si es increada, es Dios, o, por el contrario, significa que Dios no es la única sustancia increada. En cualquiera de las posibilidades, se llegaría a conclusiones absurdas o impías, especialmente al identificar espacio y Dios (lo cual, como se adivina, conecta con el aspecto teológico del problema, que trataremos en el siguiente apartado).

16 Por otra parte, la crítica del principio 'átomos y vacío', caracteristico del atomismo, se apoya en varias citas clásicas. Se acude a Aristóteles (en "Leucippe", 204a y 205a), a la refutación de Maimónides dirigida a los atomistas árabes (en "Leucippe", 203 y 206a), a la resefia de Plutarco sobre las opiniones clásicas -mayoritariamente contrarias al vacío- (en "Leucippe", 204a). 
Desarrollado el argumento, Bayle sin embargo concede que no todos han aceptado el esquema 'sustancia / accidente', y cita a Gassendi y a Locke - y ha de recordarse que este rechazo ya fue formulado por otros 17 . Pero ese intento por escapar al esquema no lo toma Bayle como una salida airosa al problema. Entiende que esa vía renuncia a una definición precisa del espacio (vacío), lo cual sería un modo de rehusar el problema pero no de resolverlo. Para ilustrarlo, Bayle reproduce el siguiente pasaje de Locke:

Si l'on demande, comme on accoutume de faire, si l'espace sans corps est substance ou accident, je répondrai sans hésiter que je n'en sais rien; et je n'aurai point d'honte d'avouer mon ignorance, jusqu'à ce que ceux qui font cette question me donne une idée claire et distincte de ce qu'on nomme substance ${ }^{18}$.

Esta confesión de ignorancia por parte de Locke, al igual que otras dificultades argumentadas por Bayle contra la idea de una extensión sin cuerpo ${ }^{19}$, conduce a la moraleja que quiere extraer Bayle. Esta moraleja, como se verá, no consiste en la defensa del punto de vista tradicional del espacio como concepto inextricablemente ligado a la extensión efectiva de los cuerpos, sino en el mostrar las contradicciones en que se incurre en cualquiera de los presupuestos, una contradicción por ejemplo que ya estaría inscrita en el propio concepto de extensión ${ }^{20}$.

17 Gassendi y Locke no son los primeros en rechazar el dilema 'substancia / accidente' aplicado al espacio. Antes lo hicieron Patrizzi y Bruno; y también hallamos el mismo rechazo en Pascal y Guericke.

18 "Zénon", 55b-56a. La cita corresponde a John LOCKE, Essay on human understanding (1690), II, XIII, \$17.

19 Esas otras dificultades las argumenta Bayle recordando varias de las refutaciones aducidas frente a la pretensión de correlacionar la divinidad con la extensión no material, o frente a la idea -extraída de la tradición escolástica- de considerar el espacio como privación, propiamente como nada. Respecto al primer punto, Bayle (cf. "Zénon", 54 b y 55b) recuerda la polémica de Arnauld con Malebranche, y la polémica entre Petit y De la Chambre: no habría modo de compatibilizar la extensión con el espíritu. Respecto al segundo punto, Bayle reproduce (cf. "Zénon", 54b-55a) el argumento de Hartsoeker contra el vacio, si por vacio entendemos la privación, la nada.

20 Esta contradicción intrínseca al concepto de extensión la desarrolla Bayle en la nota $G$ de "Zénon", 41-49, cuyo contenido resume así: "Il [Zénon] argumentait avec vigueur contre l'existence du mouvement. Quelques unes de ces objections là-dessus nous ont été conservées dans les écrits d'Aristote; mais il est vraisemblable qu'il en proposait plusieurs autres, qui étaient peutêtre les mêmes que l'on verra ci-dessous, et dont quelques-unes combattent l'existence de l'étendue" (31a). 
Para mejor conducir a esta moraleja, Bayle despliega otra línea argumentativa, la que examina la cuestión del movimiento y el vacío - segunda de las cuestiones anteriormente anunciadas-. En efecto, Bayle recuerda el argumento formulado por Meliso, que el movimiento implica el vacío, argumento que fue retomado por los atomistas y convertido así en un enunciado recurrente a favor de la posición vacuista. Pero, como recuerda Bayle, el sentido original de la proposición de Meliso es la de negar el movimiento, pues entiende que el vacío es un absurdo. Esa imposibilidad del movimiento sería también una opinión compartida por Zenón al exponer sus famosas aporías sobre el movimiento.

Así pues, de la confluencia de ambos argumentos se llegaría a conclusiones muy dificultosas: si hay, como parece, movimiento, debe aceptarse el vacío; pero si no se admite el vacío, no podrá admitirse el movimiento. Y Bayle, ante esta alternativa imposible, propone una salida:

Ne concluez point, de l'impossibilité du mouvement dans le plein, qu'il y a du vide; concluez plutôt de l'impossibilité du vide qu'il n'y a point de mouvement, c'est-d-dire, de mouvement réel; mais tout au plus une apparence de mouvement, ou un mouvement idéal et intelligible ${ }^{21}$.

Esta negación del movimiento "real" lleva, pues, a afirmar tan sólo un movimiento "ideal e inteligible". Tal conclusión forma parte de la moraleja que persigue Bayle. Y la desarrolla en tres corolarios que siguen al pasaje ahora citado. En el primero reivindica una cierta acatalepsia (o incomprensibilidad de las cosas), una acatalepsia que alcanza a los conceptos de extensión, de movimiento, de sustancia, de materia 22 . En el segundo corolario, se dice en passant que la hipótesis del vacío arruina la doctrina de la sustancia única de Spinoza 23. Y el tercer corolario -el más importante- explicita la conclusión ya apuntada antes:

La dernière conséquence que je veux tirer est que les disputes du vide ont fourni une raison spécieuse de nier que l'étendue ait une existence réelle hors de notre entendement ${ }^{24}$.

21 “Zénon”, 56a.

22 En este primer corolario (cfr. "Zénon", 56ab), por otra parte, se recuerda otra vez que los mathématiciens se inclinan por el vacío, al admitir la evidencia del movimiento y el propio argumento de Meliso. Pero se recuerda el caso de algunos mathematiciens que rechazan el vacio, citando a Leibniz y De Volder.

23 Cfr. "Zénon", 56b-57a.

24 "Zénon", 57a. 
Y se concluye también así:

Or si la nature de l'étendue pénétrable [vacía] ou impénétrable [plena] entraîne avec soi de si grands inconvéniens, le plus court est de dire qu'elle ne peut exister que dans notre esprit ${ }^{25}$.

Así pues, la moraleja a que nos conduce Bayle es el afirmar que el problema del espacio ha de remitirse al entendimiento. Esta conclusión aclara lo ya dicho sobre el espacio vacío como "idole" 26 - y ese es el tercer punto a que hacíamos referencia- Con este término se quiere subrayar que el espacio no tiene una realidad efectiva, sino que es una apariencia, o una construcción mental. Por eso dice Bayle que la aceptación por parte de los mathématiciens del espacio vacío, aún comprobando que es una opinión ganadora, es como un nuevo ídolo moderno.

En esa crítica, Bayle quiere apoyarse en Locke, al que cita repetidamente en este artículo "Zénon" 27 . Y es cierto que la elaboración de Locke consiste en concebir o deducir la idea de espacio a partir de lo que el entendimiento puede comprender, no a partir de consideraciones científicas o empíricas ${ }^{28}$. En ese sentido, la concepción de Locke apoya la pretensión de Bayle de abordar el problema del espacio por remisión al entendimiento; pero no tiene razón Bayle al dar a entender que Locke es contrario al vacío ${ }^{29}$.

Salvando, pues, este error de Bayle —más que error, es una interpretación forzada-, lo cierto es que la indicación de Bayle tiene suma importancia. Da fe de un nuevo modo de abordar el problema del espacio, modo que está al margen de los antecedentes clásicos y de alguna forma al margen de la tradición

25 "Zénon", 57b. Esta frase cierra el tercer corolario de la nota I.

26 Recuérdese el encabezamiento de la nota $G$ del artículo "Leucippe": "Le vide... devient l'idole favorite des plus célèbres mathématiciens" (en 204a).

27 Las referencias de Bayle a Locke se encuentran en el artículo "Zénon” (en 54b, 55b, 56a, 56b). Hay otras dos referencias a Locke en el Dictionnaire, pero no tienen que ver con esta cuestión (ver la entrada Locke del índice temático).

28 Por ejemplo en Essay, II, XIII, $\$ 24$, LocKE afirma que es posible formarnos la idea de un espacio distinta a la idea de cuerpo.

29 LOCKE dice: "Porque el vacío, independientemente de que afirmemos o neguemos su existencia, significa espacio sin cuerpo, y su existencia nadie la puede negar como posible, a no ser aquellos que quieren hacer infinita a la materia, y quitar a Dios el poder de aniquilar cualquier partícula de ella" (Essay, II, XIII, conclusión del $\$ 22$; la alusión última de la frase se dirige a Descartes). Y en otro lugar dice que "no es necesario probar la existencia real del vacío, sino la de la idea del mismo" (Essay, II, XIII, \$24). 
científico-empírica. Es la citada remisión al entendimiento del problema; y esta línea de reflexión es la que identificamos en importantes filósofos: está en Hobbes, en Locke, en Leibniz a su modo, y luego en Hume y en Kant. Ante este aspecto común, poco importa incluso que Locke se desvíe de la crítica al vacío que realizan los restantes; todos ellos coinciden en vincular el problema del espacio al entendimiento, en no afirmar una realidad objetiva para este espacio, y en aceptar que éste es sobre todo un orden geométrico, es un orden que el entendimiento impone en la asimilación de la disposición de las cosas percibidas.

Calificar de "ídolo" o "fantasma" a ese espacio es la conclusión lógica, sobre todo si lo que se quiere es subrayar que la pretendida idea de un espacio objetivo es una ilusión. Esos calificativos también los utilizan Hobbes y Leibniz, por ejemplo, con la misma intención ${ }^{30}$; y sabemos que Hume se apoyó en este artículo "Zénon" del Dictionnaire de Bayle para desarrollar su teoría del espacio ${ }^{31}$.

Así pues, este aspecto del examen crítico realizado por Bayle tiene una evidente continuidad en el debate filosófico futuro.

\section{El problema teológico del espacio}

Este aspecto teológico no será tan debatido en el futuro, como lo fue el anteriormente reseñado; pero, en cambio, tiene mayor impacto en la época específica del Dictionnaire. En tal sentido, las indicaciones de Bayle al respecto son muy representativas de las discusiones del momento.

30 Hobbes concibe el espacio como "fantasma". Así lo dice en su De Corpore. "Space [... ] is nothing but a phantasm, in the mind or the memory, of a body of such magnitude and such figure" (Thomas HoBbes, The English Works, vol. I, p. 106). LeIBNIZ, por su parte, denuncia en el contexto de su polémica con Clarke la pretensión de un espacio absoluto y vacío, con estas palabras: “ [... ] l'Espace qui est Idolum Tribus de plusieurs, comme parle Verulamius, n'est plus une substance, ny un etre absolu, mais un ordre comme le temps" (Carta de Leibniz a Rémond de 27 marzo 1716, en André RoBinet (ed.), Correspondance Leibniz-Clarke, París, 1957, p. 61). Similares términos en el $\$ 14$ del Escrito $44^{\circ}$ de Leibniz de la polémica (cfr. Robinet, p. 89).

31 Esa teoria del espacio de David Hume se halla en su Treatise of Human nature, lib. I, parte II (De las ideas de espacio y tiempo), especialmente sección 5. Sobre la influencia de Bayle (especialmente sus artículos "Spinoza" y "Zénon" del Dictionnaire) en Hume, véase: N. Kemp SMITH, The philososophy of David Hume: a critical study of its origins and central doctrines (Macmillan, Londres, 1941), pp. 506-516; J. P. PITTION, "Humes's reading of Bayle: an inquiry into the source and role of the Memoranda", Journal of the History of Philosophy, 15 (1977), pp. 373386. 
Como ya se dijo, el índice temático del Dictionnaire en sus dos entradas referidas al espacio retiene este aspecto teológico del problema, enunciando concretamente dos cuestiones: la cuestión de la correlación entre el espacio y la inmensidad divina; y la cuestión del llamado espacio "imaginario". La primera aparece en el artículo "Zénon"; la segunda en el artículo "Francus".

Respecto a la primera, el contexto de la discusión de Bayle es aquel ya comentado acerca de la aplicación del esquema 'sustancia / accidente' al problema del espacio. Ahí Bayle deducía la inconsistencia de cualquiera de las alternativas; y explicaba en particular que la opción del espacio como sustancia increada conducía a la confusión entre espacio y Dios, calificando tal solución de impía. El argumento es que siendo el espacio divisible en partes, tal confusión implicaba que Dios también sería divisible, o sea múltiple, lo cual contradice uno de los atributos básicos de la divinidad, esto es, su Unidad o Simplicidad $^{32}$.

Esta discusión seguía en Bayle con la reseña de algunas de las refutaciones formuladas contra la pretensión de hacer de Dios el correlato de la extensión inmaterial, por ejemplo reproduciendo este pasaje de Arnauld:

Tant s'en faut que la simplicité de Dieu nous puisse donner lieu de croire qu'il peut être étendue, que tous les théologiens ont reconnu après saint Thomas que c'était une suite nécessaire de la simplicité de Dieu de ne pouvoir être étendue 33 .

Como se ve, el argumento es simple: extensión y simplicidad de la divinidad son incompatibles. Con ello, Bayle retoma una cuestión que no era nueva; pues, de hecho, una larga discusión teológica venía manteniéndose desde la Edad Media a propósito de cómo interpretar el atributo clásico de la ubicuidad de Dios, si con un claro correlato espacial o no. Y en este punto debe advertirse que la afirmación de Arnauld —asumida por Bayle — sobre "tous les théologiens" apoyando esa tesis de la incompatibilidad no es una afirmación exacta, pues no todos los teólogos la compartían - y de recordarlo se encarga, como se verá, el propio Bayle al reseñar la opinión de Suárez-.

32 Cfr. "Zénon", 54a. Sobre las concepciones aceca de la divisibilidad de la extensión, véase Philip Cummins, "Bayle, Leibniz, Hume and Reid on Extension, Composites and Simples", History of Philosophy Quarterly, 7 (1990), pp. 299-314.

33 "Zénon", 54b, reproduciendo un pasaje de Antoine ARNAuld, Défense contre la Réponse au livre des vrais et des fausses Idées. 
Lo importante a retener de estos apuntes de Bayle es el constatar cómo ese aspecto teológico del problema estaba muy vivo en la época del Dictionnaire y en sus inmediatos antecedentes. Baste recordar que el argumento sobre la multiplicidad del espacio-extensión y la imposible multiplicidad-divisibilidad de la divinidad fue tema importante en la polémica entre More y Descartes: Descartes negó en base a ese argumento la correlación entre la extensión y la divinidad; y More, en cambio, aceptó que las sustancias espirituales fueran res exten$s a^{34}$. Igualmente, Pascal en su polémica con Nöel acerca del espacio vacío recuerda que en el bando de los plenistas estarían los herederos vergonzantes de la idea de la plenitud basada en la correlación con la inmensidad divina. Y en la explicación de Pascal es claramente perceptible la sospecha teológica que recae sobre esos defensores de la "inmensidad divina" 35.

En suma, esa idea de la inmensidad divina correlacionada con el espacio, tanto en la opción de la plenitud como en la de la vacuidad, es una idea muy discutible y discutida. Por eso, tal como nos reseña el propio Bayle, la opinión más moderna a favor de la concepción del espacio como entidad independiente y conceptualmente vacía -o sea, la opinión de Gassendi y Locke, por ejemplo-, renuncia a ese ámbito de la discusión: renuncia al esquema 'sustancia / accidente'; se niega a evaluar el concepto de espacio en los términos de la alternativa entre sustancias corporales y sustancias espirituales; y se resiste a plegarse a la opinión de tradición escolástica que toma el espacio vacío de cuerpo como concepto privativo, el espacio propiamente como nada ${ }^{36}$.

Pero Bayle considera que estas renuncias no son consistentes, pues entiende que significan una renuncia a ideas claras y distintas, algo que supondría alinearse con el pirronismo. Bayle puede comprender que los mathématiciens afirmen la evidencia del espacio vacío, dejando de lado las discusiones tradicio-

34 La discusión puede seguirse en Alexandre KoYRé, From the closed World to the infinite Universe (Baltimore, 1957), cap. V.

35 Decía Pascal en su carta a Le Pailleur de 1648 (a propósito de su polémica con Noël), resumiendo las diversas posiciones plenistas: "unos abogan por el aether y excluyen cualquier otra materia; otros, por los espíritus del líquido, en detrimento del aether, otros, apelan al aire alojado en los poros del cristal, y nada más; otros, al aire rarificado y vacío de cualquier otro cuerpo; ha habido,por último, quienes no atreviéndose a postular la inmensidad de Dios han elegido a un hombre lo bastante ilustre por nacimiento y méritos para poner su espíritu y hacerle que llene todas las cosas" (Blaise PASCAL, Tratados de preumática, ed. A. Elena, Alianza, Madrid, 1984, p. 102). La alusión irónica se dirige a Descartes.

36 Cfr. "Zénon", 54b y 55b. 
nales; pero él quiere hurgar en sus inconsistencias, al fin y al cabo para conducirnos a las moralejas que ya hemos comentado: la acatalepsia, la remisión al entendimiento del problema del espacio.

Así pues, el argumento teológico será un aspecto en el que insiste Bayle. Otra de sus facetas es la ya enunciada cuestión de los espacios "imaginarios". Esta aparece en el artículo "Francus" — como ya se dijo-, y también en el artículo "Leucippe" 37.

Por espacio "imaginario" hay que entender el calificativo, acuñado en la Edad Media y adoptado por la tradición escolástica, que se aplica a los espacios de la región extracósmica, o sea el espacio situado más allá del Firmamento o límite del mundo. Ese espacio, por definición, no contiene cuerpos; y tendría, pues, una entidad distinta a la del espacio intracósmico - éste sí ocupado por cuerpos y de carácter dimensional-. Según el esquema cosmológico aristotélico, no tendría sentido preguntarse por ese espacio extracósmico; pero en la Edad Media ya se producen los primeros abandonos del esquema aristotélico. Thomas Bradwardine, por ejemplo, se refiere a ese espacio extracósmico llamándolo "vacío imaginario infinito" 38 . Y afirma que la inmensidad divina, correlato de uno de los atributos básicos de la divinidad, la infinitud, está presente en él.

Como observa Bayle, esa apelación a los espacios imaginarios como equivalente del vacío infinito extracósmico viene a ser la reedición cristiana de la posición cosmológica estoica, frente a la aristotélica ${ }^{39}$; una reedición que pone el acento en la citada correlación entre espacio imaginario e inmensidad divina. Aunque ciertamente, esta opinión - como dice Bayle- "está sujeta a mil dificultades" 40 . Pues, en efecto, ya de la Edad Media arranca una diversidad de in-

37 Cfr. "Francus", 588-589; "Leucippe", 204b (esta referencia a "Leucippe" no aparece en el índice temático relativo a Espaces imaginaires).

38 Thomas Bradwardine, De causa Dei, lib. I, cap. V, p. 177. La obra fue compuesta en 1344 y publicada en 1618 por Henry Savile en Oxford (reimpr. facs. Frankfurt, 1964). Sobre Bradwardine, consúltese: Edward Grant, Much ado about nothing. Theories of space and vacuum from Middle Ages to the Scientific Revolution (Cambridge Univ. Press, 1981), pp. 135-147; Alexandre KoYRE, "Le vide et l'espace infini au XIVe siècle", en Etudes d'histoire de la pensée philosophique (Gallimard, París, 1971), pp. 37-92. Sobre el concepto en general de espacio imaginario, ver la citada obra de. Grant: en pp. 117-121 describe los origenes y el desarrollo de la expresión "espacio imagiriario"; luego en cap. 7, pp. 148-181, describe el impacto de este concepto de la tradición escolástica en los siglos XVı y XVII.

39 Cfr. "Leucippe", 204b

40 "Francus", $288 \mathrm{ab}$. 
terpretaciones acerca del atributo de ubicuidad de Dios: unos, como Bradwardine, lo interpretan en términos directamente espaciales; pero otros, como Santo Tomás de Aquino o Duns Scoto rechazan esa correlación espacial. El eco de esa polémica todavía está vivo en los siglos XVI y XVII. Así, Bayle cita a Suárez como autoridad representativa de la posición favorable a la idea de la presencia espacial de Dios en los espacios "imaginarios", de hecho en la totalidad del espacio. Y Bayle trae de nuevo a colación la réplica de los cartesianos, de Arnauld, haciendo extensiva esta crítica a Malebranche ${ }^{41}$.

Lo que se ventila tras esta reseña de Bayle es lo vivo de una polémica acerca del correlato entre espacio y divinidad. Ese correlato tiene en la ambigua expresión de "espacio imaginario" su fuente de autoridad, autoridad todavía vigente a través de teólogos como Suárez. Y sabemos que está vigencia todavía será perceptible en la polémica entre Leibniz y Clarke ${ }^{42}$. De hecho, el problema de fondo es, como dice Arnauld y recoge Bayle, la incompatibilidad o no de las sustancias espirituales con el carácter extenso. Este problema se aplica tanto a la cuestión de la correlación entre espacio y divinidad, como a la cuestión de la presencia extensa y espacial del alma en el cuerpo, cuestiones ambas cruciales en las discusiones contemporáneas y antecedentes al Dictionnaire ${ }^{43}$.

Y más allá de este empeño de Bayle por mostrar las dificultades en que se incurre a partir de la consideración del problema del espacio en términos teológicos, está la intención crítica hacia Spinoza, otro de los fantasmas a batir. La exposición de Bayle se dirige sobre todo contra la hipótesis de un espacio considerado como entidad independiente, o sea básicamente la hipótesis de un espacio vacío; pero su crítica se dirige también al caso opuesto, el de la plenitud,

41 Cfr. "Francus", 588b-589a.

42 Las principales referencias al espacio "imaginario" en la polémica Leibniz-Clarke son éstas: $\$ 2$ de la Respuesta 3..$^{2}$ de Clarke; $\$ 7$ y $\$ 14$ del Escrito $4 .^{\circ}$ de Leibniz; $\$ 7$ de la Respuesta $4 .^{2}$ de Clarke; $\$ 33-35$ y $\$ 38$ del Escrito $50^{\circ}$ de Leibniz.

43 Este paralelismo con la cuestión del alma / cuerpo justifica la inclusión de esta discusión sobre los espacios imaginarios en el artículo "Francus". Bayle establece que la doctrina de Server, acerca de la presencia extensa del alma en el cuerpo, fue adoptada por Franck; idea paralela a la presencia de Dios en toda porción extensa; lo cual conduciría a una especie de deificación de toda porción creada, deificación del ser humano y deificación de cualquier trozo de mármol - dirá Bayle por ejemplo (cfr. "Francus", 588b) -; lo cual conduciría a la impiedad y a la idolatría. Por otra parte, como se sabe, también en la polémica Leibniz-Clarke la discusión establece continuos paralelismos entre el problema del espacio y el problema de la comunicación y presencia del alma con el cuerpo. 
cuando esta plenitud es interpretada como correlacionada con la divinidad. Y ése es el caso de Spinoza 44 .

En efecto, Bayle en la nota $\mathrm{N}$ del artículo "Spinoza" critica la doctrina de la sustancia única, resumida por Bayle así:

Il suppose qu'il n'y a qu'une substance dans la nature, et que cette substance unique est douée d'une infinité d'attributs, entre autres de l'étendue et de la pensée. Ensuite de quoi il assure que tous les corps qui se trouvent dans l'univers sont des modifications de cette substance, en tant qu'étendue... ${ }^{45}$

La sustancia única - Dios- no difiere, pues, de las cosas; los cuerpos serían modos de la extensión de esa sustancia. Con lo cual, se llega a otra manera de postular la identidad entre espacio e inmensidad divina, en este caso bajo el postulado de la plenitud - pues, hay que recordarlo, Spinoza es radicalmente contrario al vacío- La refutación de la doctrina de la sustancia única por Bayle se extiende en toda la nota $\mathrm{N}$, dividida en seis apartados, siendo el II el dedicado específicamente a la cuestión de la extensión ${ }^{46}$. El argumento ya es conocido: atribuir extensión a Dios implica que la división en partes de la extensión se proyecta en una similar división de la divinidad, lo cual contradice los atributos de unidad y simplicidad. Es cierto que los espinosistas replican - tal como recoge Bayle - que al no haber vacío no serían partes separadas, que un continuum asegura la unidad; pero Bayle replica que esa extensión, siendo material, arrastra consigo la mutabilidad de la materia, con lo cual el Dios de los espinosistas sería mutable.

En suma, el examen de la cuestión teológica del espacio en cualquiera de sus modalidades, espinositas o no, conduce para Bayle a un mismo resultado. Se trata de rechazar cualquiera de las formulaciones que pretendan establecer una correlación entre la divinidad y el espacio.

44 La doctrina de Spinoza es, como señala el propio Bayle, una reedición de la vieja doctrina del alma del mundo. Véase al respecto: Jean-Charles DARMON, "Gassendi contre Spinoza selon Bayle: ricochets de la critique de l'âme du monde", Archives de Philosophie, 57 (1994), p. 523-540.

45 "Spinoza", 438b. La nota $\mathrm{N}$ del artículo (La plus monstrueuse hypothèse... la plus diamétralement opposée aux notions les plus évidentes de notre esprit), en pp. 438-446.

46 Cfr. "Spinoza", 440b-441b. 


\section{Resumen y conclusiones}

Hemos tomado el Dictionnaire historique et critique de Bayle fundamentalmente como un testimonio de la época. Nos interesa en este caso a modo de ilustración del debate de ideas en torno a la cuestión del espacio. El Dictionnaire, a pesar del sesgo que inevitablemente introduce su autor, se nos presenta, pues, como un notario que levanta acta de las concepciones en presencia.

En un rápido resumen, diremos que estas concepciones son básicamente de tres tipos: la opinión de filiación atomista (vía Gassendi) que admite un espacio independiente de los cuerpos y, por lo tanto, vacío; la opinión de filiación cartesiana que niega tal independencia y considera el espacio - mejor llamarlo extensión- como cualidad intrínseca a la materia (llamada entonces res extensa); y la opinión que hace de la remisión al entendimiento la base sobre la que elaborar la idea de espacio (representada en este caso por Locke).

A estas tres opiniones, que podemos encuadrar en un contexto científico-filosófico, debe añadirse la incidencia de toda una discusión teológica directamente relacionada con el problema. Al fin y al cabo, estamos situados en una época de encrucijada e inflexión: encrucijada entre las objeciones clásicas contra el espacio vacío y la moderna aceptación del vacío; inflexión entre una consideración en términos metafísicos y teológicos y una consideración en términos científicos y geométricos. Espacio lleno o vacío, espacio relativo o absoluto, serían dos modos de nombrar ese hiato. O dicho de otro modo, el Dictionnaire nos permite observar la incidencia de los dos factores que intervienen en el proceso de conceptualización del espacio moderno: el factor geometrizador, y el factor divinizante.

Los contenidos del Dictionnaire los hemos clasificado en tres apartados, el científico, el filosófico, y el teológico, aunque advirtiendo que los tres a menudo se entremezclan.

En cuanto al primero, el aspecto científico, hemos destacado la poca atención que Bayle presta a las cuestiones experimentales. Hay unas pocas referencias a los experimentos barométricos de Torricelli y de Pascal, sólo para admitir que esos experimentos sirvieron para desbancar el viejo principio del horror vacui, pero a la vez destacando que los plenistas de inspiración cartesiana podían interpretarlos también en clave plenista. Los experimentos no aportarían, pues, un criterio definitivo acerca del vacío y del espacio. Y es que la discusión que interesa a Bayle no es la experimental, sino la discusión entre sistemas. Pa- 
ra ésta, Bayle resume que la concepción de los mathématiciens de un espacio vacío está en boga frente a la concepción de Descartes. Y cita a Gassendi, a Huygens y a Newton como ejemplos de tales mathématiciens.

Pero esta solución no satisface a Bayle: califica como "ídolo" a ese espacio defendido por los científicos. De ahí que su examen se extienda bastante en el aspecto filósofico — segundo de los apartados a que nos referíamos-. Para este examen, Bayle repasa las doctrinas clásicas del atomismo, retoma ampliamente el problema 'sustancia / accidente' aplicado al espacio, discute el viejo argumento de que el movimiento implica el vacío. Como conclusión de este repaso exhaustivo cabe señalar que la intención perseguida por Bayle es la de mostrar las insalvables dificultades en que se incurre en cualquier alternativa, sea átomos o continuum, sea el espacio modo o accidente, sea aceptando el movimiento o no. El inspirador de esta argumentación es el aquí obviamente Zenón de Elea (el examen de Bayle, en efecto, se produce principalmente en el artículo "Zénon").

La conclusión de Bayle podría resumirse en estos pasajes:

La dernière conséquence que je veux tirer est que les disputes du vide ont fourni une raison spécieuse de nier que l'étendue ait une existence réelle hors de notre entendement ${ }^{47}$.

Or si la nature de l'étendue pénétrable [vacía] ou impénétrable [plena] entraîne avec soi de si grands inconvéniens, le plus court est de dire qu'elle ne peut exister que dans notre esprit ${ }^{48}$.

Así pues, la cuestión del espacio es remitida al entendimiento. $Y$ como ya hemos dicho, llamar "ídolo" al espacio es un modo de subrayar que la pretendida idea de un espacio objetivo, espacio al margen de nuestro entendimiento, es una ilusión. Esta es una importante conclusión, que está en la línea de las reflexiones de Hobbes, de Locke (al que cita repetidamente Bayle), de Hume y de Kant.

A parecidas conclusiones se llega al analizar el problema teológico del espacio - tercero de los contenidos a que hacíamos referencia-. La dificultad es aquí la que deriva de considerar identificables o correlacionables el espacio y la

47 "Zénon", 57a.

48 "Zénon", 57b. Esta frase cierra el tercer corolario de la nota I. 
inmensidad divina. Bayle muestra que la divisibilidad de la extensión se aplicaría en este caso a la divinidad, desmintiendo por tanto los atributos de unidad y simplicidad inherentes a tal divinidad. Sin embargo, la explicación de Bayle testifica que la opinión favorable a la identificación entre espacio e inmensidad divina es una opinión que cuenta con sus partidarios: como él mismo señala, algunos teólogos - como Suárez - la avalarían, y sus antecedentes se remontan a la discusión del llamado espacio "imaginario".

La intención de Bayle no sólo se limita al caso del espacio diferenciado del cuerpo (o sea, el espacio vacío); también se aplica a toda consideración que haga compatible la extensión con las sustancias espirituales, aunque sea con un espacio pleno. Es decir, tanto en la vacuidad como en la plenitud, el problema reside en dicha correlación entre extensión y espíritu. Problema éste que Bayle aplica a la crítica de la doctrina de la sustancia única de Spinoza, sustancia única -Dios- cuyos modos serían, entre otros, la extensión y la materia misma.

La conclusión es la ya repetida: la confusión entre espacio y divinidad, sea en el espinosismo o sea en el vacuismo, es una opinión no admisible y sujeta a mil dificultades.

De todas estas consideraciones de Bayle y de todas sus referencias a los términos de la discusión en esa época, cabe deducir que su principal intención es la de destacar que toda pretensión de considerar el espacio como una realidad objetiva incurre en serias contradicciones. Por eso, se inclina por una consideración en términos de remisión al entendimiento: el espacio sólo sería comprensible desde esa apelación al sujeto. Esta opinión la toma, entre otros, de Locke, y, como se sabe, es una opinión que alimenta toda una línea de la reflexión filosófica acerca del espacio: es la que va de Hobbes a Locke, a Hume y a Kant.

Pero junto a la constatación de la opción personal de Bayle, su Dictionnai$r e$ nos suministra además una valiosa información sobre las distintas opciones en presencia. Dicho resumidamente: esa información nos permite confirmar que en el proceso que conduce a la conceptualización moderna del espacio intervienen un factor geometrizador y otro divinizante. Por conceptualización moderna entendemos la consideración de un espacio real al margen de los cuerpos, espacio que puede estar vacante de materia y espacio como realidad geométrica. Este espacio es el suministrado por la cosmología newtoniana, pero depurado de sus connotaciones metafísicas una vez la victoria newtoniana haya alcanzado al Continente gracias a divulgadores como Voltaire. 
Esa moderna conceptualización del espacio se "olvidará" de esas connotaciones de origen, especialmente de aquella famosa consideración de Newton del espacio como sensorio de Dios ${ }^{49}$. Se olvidará, pues, del factor divinizante. Este factor es incuestionable en el caso de Newton, pero también lo es más allá de Newton -como lo demuestra Bayle al subrayar lo vivo de una discusión en torno a la cuestión de la inmensidad de Dios confundida con el espacio-. Pero además, el Dictionnaire testifica con claridad la presencia del factor geometrizador en las concepciones del espacio. Este factor está presente a través de los llamados mathématiciens (y entre ellos, Bayle cita al propio Newton); y a través del propio Locke, pues la opción de Locke no deja de ser una reificación de la intuición geométrica.

La moderna conceptualización del espacio se apoya en todos estos antecedentes, o sea en los dos factores, la divinización y la geometrización del espacio, en una mezcla compleja y a menudo conflictiva. El Dictionnaire de Bayle da fe de esta complejidad 50 .

49 Esta consideración de Newton acerca del espacio como sensorio de Dios aparece en la cuestión 20 de la $2 .^{2}$ ed. (latina, 1706) de su Optica - luego, será la Cuestión 28 en las siguientes ediciones-. No aparecía en la $1 .^{a}$ ed. de 1704.

50 Sirva esta conclusión para matizar la opinión de Grant, opinión a nuestro entender excesivamente unilateral al destacar sólo el valor del factor divinizante. Nos referimos concretamente a su obra ya citada Much ado about nothing, p. 232-234 (en el apartado "Was space 'geometrized' in the seventeenth century?"), donde Grant criticaba la interpretación de Koyré consistente en describir el paso a la concepción moderna del espacio como el paso desde la concepción aristotélica (un conjunto ordenado de lugares) a la concepción geométrica (una extensión esencialmente infinita y homogénea). Cfr. Alexandre KoYRE, From the closed World to the infinite Universe (Baltimore, 1957), p. viii. Grant opina que el aspecto relevante es el proceso de la divinización del espacio, no el de su geometrización. Nosotros creemos que los dos factores son igualmente importantes y no tienen porqué excluirse. 